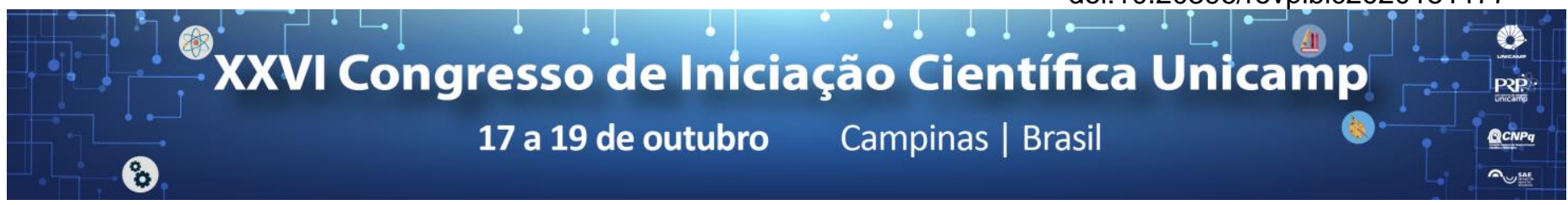

\title{
Construction and assessment of a low cost NDVI sensor
}

\section{Rafaella Pironato Amaro*, Felipe Ferreira Bocca, Luiz Henrique Antunes Rodrigues.}

\begin{abstract}
Given the potential of low cost sensors for agriculture, a monitoring system with low cost components was constructed to evaluate its capacity to detect variations in vegetative vigor in smooth lettuce seedlings under different growing conditions. The results showed temporal inconsistency and low variability in NDVI values. The inconsistency can be attributed mainly to factors such as luminosity and the high capacity of retention of water and nutrients of the substrate. Days with milder light intensity produced potentially better NDVI values. The low variability of NDVI eventually contributed to its low correlation with mass.
\end{abstract}

\section{Key words:}

Open hardware sensors, Raspberry, Plant water stress monitoring.

\section{Introduction}

Spectral sensors allow monitoring of plant stress and its nutritional status ${ }^{1}$, in addition to showing the growth and development of plants along the phenological stages.

The objective of this work was to evaluate the capacity of a plant monitoring system with low cost components to detect variability in vegetative vigor.

\section{Results and Discussion}

The low cost camera (Pi NolR - Raspberry Pi 2 model B) was associated with a filter (Roscolux 2007 Storaro Blue) for data acquisition that allowed the monitoring of 30 smooth lettuce seedlings (Lactuca sativa L.) and obtaining NDVI in images under different growth conditions.

The five forms of treatment used were: $30 \mathrm{ml}$ of nutrient solution and water, $15 \mathrm{ml}$ of nutritive solution and water, $30 \mathrm{ml}$ of only water, $15 \mathrm{ml}$ of only water and no treatment. The NDVI general behavior in relation to the different types of treatment was inconsistent over time and with low variability. This can be attributed to the substrate that has high water retention capacity and many nutrients, which would take longer to present differences between the plants that could be captured by the camera and the temporal inconsistency can be attributed mainly to the luminosity, which was not controlled although earnings are fixed.

The camera captured the variability in lettuce leaves, since the NDVI values are lower where the plants are stressed and visually yellowish (Figure 1). (a)

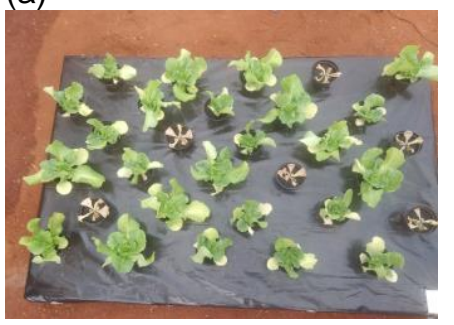

(b)

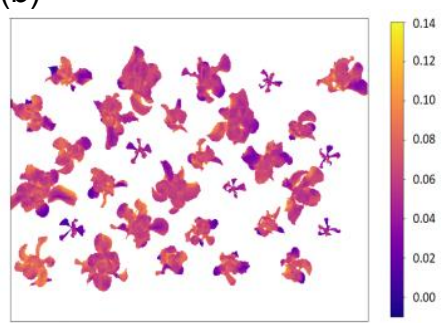

Figure 1. Photo (a) and mask of NDVI (b). (a)

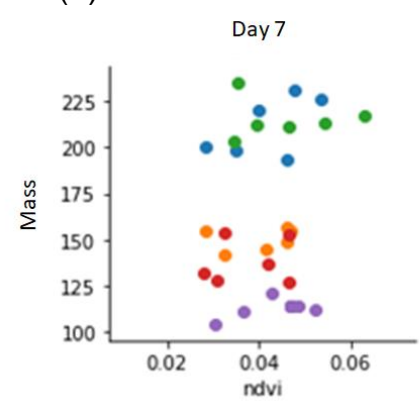

(b)

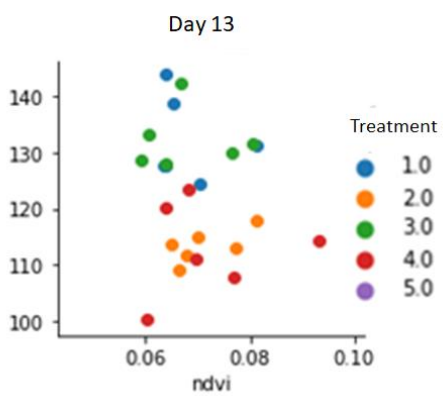

Figure 2. Correlation between plant mass and NDVI, for each plant. Image acquisition on the 7th day (a) and on the 13 th day $(b)$.

The low NDVI variability eventually contributed to its low correlation with the mass which, although not indicating growth due to interferences such as humidity, maintained grouping between the masses of treatments 1 and 3 and 1 and 4 , showing that the nutrient solution used was not enough to distinguish the treatments.

\section{Conclusions}

The general behavior of the NDVI was inconsistent temporally and with low variability due to the substrate, due to its high retention capacity of water and many nutrients, and also to the luminosity that was not controlled.

Acquiring images in the morning or isolating the effect of brightness, can lead to more stable results for NDVI.

Identifying the variability of each plant on a daily basis rather than temporary basis seemed to be more interesting as long as it is possible to find the best camera configuration by optimizing gain and brightness for that acquisition day.

In order to avoid external interferences in the plant mass measurements, a protocol should be created to quantify growth by weighing.

\section{Acknowledgement}

We thank PIBIC/CNPq for the scholarship of the first author and CAPES and CNPq for the scholarships of the second author.

${ }^{1}$ SERRANO, L.; FILELLA, I.; PEÑUELAS, J. Remote Sensing of Biomass and Yield of Winter Wheat under Different Nitrogen Supplies. p. 723-731, 2000. 